\title{
Design of Multi-Octave Band GaN-HEMT Power Amplifier
}

\author{
Gulesin Eren\#, Ozlem A. Sen\#, Basar Bolukbas\#, Gokhan Kurt\#, Orkun Arican\#, Omer Cengiz\#, Sila T.K. Unal\#, \\ Yildirim Durmus\# and Ekmel Ozbay\# \\ \#Department of Electrical and Electronics Engineering, Nanotechnology Research Center, Bilkent University, 06800 Ankara, \\ Turkey
}

\begin{abstract}
This paper describes design, fabrication and measurement of $6 \mathrm{GHz}-18 \mathrm{GHz}$ monolithic microwave integrated circuit (MMIC) amplifier. The amplifier is realized as coplanar waveguide (CPW) circuit using $0.3 \mu \mathrm{m}$-gate GalliumNitride (GaN) HEMT technology. The amplifier has a small signal gain of $7 \pm 0.75 \mathrm{~dB}$. The output power at $3 \mathrm{~dB}$ compression is better than $24 \mathrm{dBm}$ with $16 \%-19 \%$ drain efficiency for the whole $6 \mathrm{GHz}-18 \mathrm{GHz}$ frequency band under continuous wave (CW) power.
\end{abstract}

Index Terms-GaN HEMT, amplifier, multi-octave bandwidth, wide-band matching networks, gain equalization networks.

\section{INTRODUCTION}

Wide-band amplifiers are important for electronic warfare, instrumentation, radar applications. Due to physical properties, Gallium-Nitride $(\mathrm{GaN})$ has become the promising material of choice for wideband power amplifiers.

Fully monolithic microwave integrated circuits realized with gallium nitride $(\mathrm{GaN})$ high electron mobility transistors are of interest to design and implement microwave power amplifiers due to its superior properties like high breakdown voltage, high current density, high thermal conductivity and high saturation current [1]. Large band gap energy and high saturation velocity of $\mathrm{AlGaN} / \mathrm{GaN}$ high electron mobility transistors (HEMTs) are attractive features for high power applications compared with the conventional material for high power applications- gallium arsenide (GaAs). Wide bandgap nature of $\mathrm{GaN}$ makes the material more advantageous than GaAs for the operations that requires high voltage, power and temperature. The high power capability of GaN enables us to make devices with smaller size than of GaAs based devices for the same output power. Thus, device impedances in $\mathrm{GaN}$ technology are higher than the GaAs technology which makes broadband matching easier [2].

In this paper, we proposed a multi-octave MMIC amplifier realized with dimensions of $3.5 \times 1.5 \mathrm{~mm}^{2}$ by using reactive matching topology. The amplifier is a coplanar single stage MMIC amplifier operating between $6 \mathrm{GHz}$ and $18 \mathrm{GHz}$ suitable for electronic warfare applications. In the first section fabrication details and performance of $\mathrm{GaN}$ transistor is summarized. Then, the design procedure of the amplifier is given. Finally measured performances of the fabricated MMICs comparing with our electromagnetic simulations are presented. The design phase was required special attention on gain flatness of small-signal response and broadband reactive matching networks over the $6-18 \mathrm{GHz}$ bandwidth. The MMIC amplifier realization in a multi-octave bandwidth was performed by using a high electron mobility transistor of $4 \times 75$ $\mu \mathrm{m}$ in coplanar technology which was processed at our own clean-room facilities.

\section{Technology And Transistor Performance}

The MMIC process has been developed using our 4-inch GaN HEMT on semi-insulating $\mathrm{SiC}$ substrate. $\mathrm{SiC}$ is used as a substrate material for this application due to its high thermal

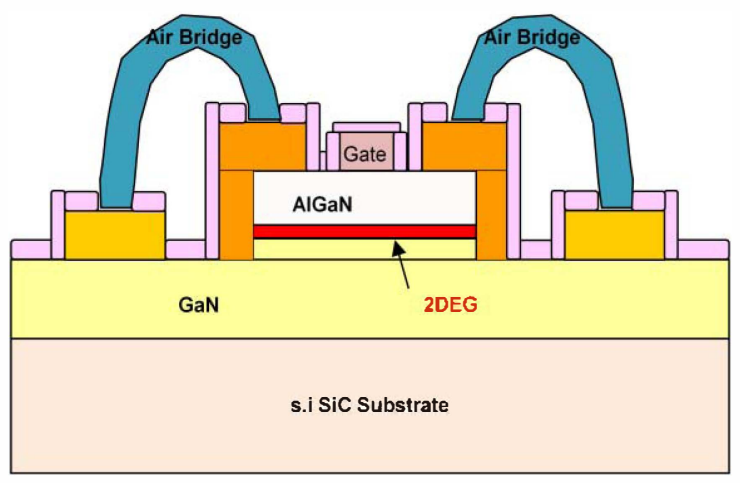

Fig. 1. Cross-sectional view of MMIC

conductivity property. The epitaxial GaN/AlGaN layers are grown on $\mathrm{SiC}$ substrate by utilizing MOCVD. Fabrication process consists of formation of Ohmic contact for drain and source connections, Schottky contact for gate metallization, $\mathrm{SiN}$ passivation and several inter-process steps. Cross sectional view of a MMIC is given in Fig. 1.

The $4 \times 75 \mu \mathrm{m}$ active device cell was characterized by DC IV, S-parameters, and load-pull characterizations. DC characterization result of $4 \times 75 \mu \mathrm{m}$ device is given in Fig. 2 .

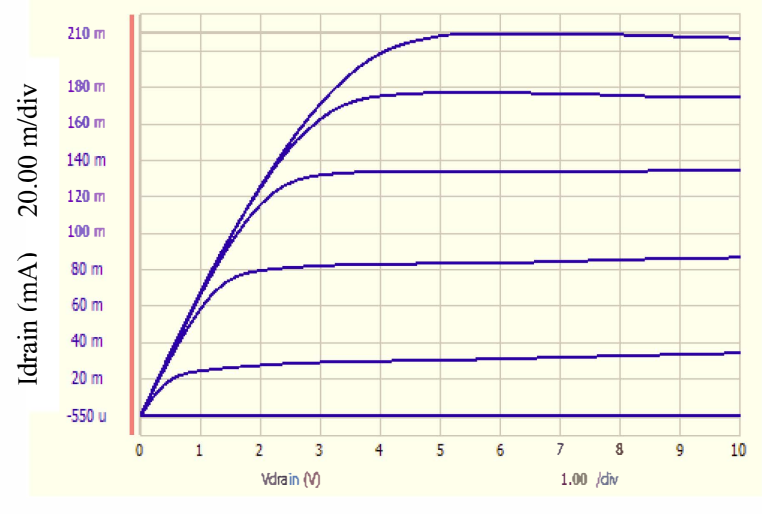

Vdrain (V) 1.00/div

Fig. 2. $I_{\text {drain }}(m A)$ vs. $V_{\text {drain }}(V)$ graph for $4 \times 75 \mu \mathrm{m}$ GaN HEMT 
$1.15 \mathrm{~A} / \mathrm{mm}$ peak drain current density, $270 \mathrm{mS} / \mathrm{mm}$ maximum transconductance, $40 \mathrm{GHz}$ of $f_{\max }$ values are achieved from the fabricated $4 \times 75 \mu \mathrm{m}$ GaN HEMT.

\section{WIDEBAND DESIGN CONSIDERATIONS}

Design process of the wide-band MMIC amplifier is described in this section. Unconditional device stabilization and broadband matching topology design require special attention. Reactive matching topology was found to be the most appropriated topology to fulfill design specifications.

Unconditional stability can be obtained by different networks. In this work adding a parallel combination of $\mathrm{R}$ and $\mathrm{C}$ in series at the input of the amplifier is chosen as stabilization network since this topology serves for both stabilization of the device and flattening the gain response by decreasing the HEMTs intrinsic gain at lower frequencies. After the stabilization realization, load-pull and source pull simulations are implemented. Table I shows the values of source and load impedances at $\mathrm{V}_{\mathrm{DS}}=15 \mathrm{~V}$, and $\mathrm{I}_{\text {Drain }}=72 \mathrm{~mA}$. Due to the choice of $\mathrm{GaN}$ technology, source and load impedances are not so far from the $50 \Omega$ system impedance and realization broadband matching would not require high impedance transformation ratio so the complexity of the wideband matching topology decreases [4].

TABLE I

\begin{tabular}{|l|l|l|}
\hline \multicolumn{3}{|c|}{ Source and Load Impedances } \\
\hline Frequency $(\mathbf{G H z})$ & \multicolumn{1}{|c|}{ Zsource } & \multicolumn{1}{c|}{ Zload } \\
\hline $6 \mathrm{GHz}$ & $45+\mathrm{j} 35.5$ & $83+\mathrm{j} 65$ \\
\hline $8 \mathrm{GHz}$ & $45+\mathrm{j} 35.5$ & $67+\mathrm{j} 75.3$ \\
\hline $10 \mathrm{GHz}$ & $46.4+\mathrm{j} 27.8$ & $67+\mathrm{j} 75.3$ \\
\hline $12 \mathrm{GHz}$ & $47.8+\mathrm{j} 20.5$ & $59+\mathrm{j} 60.4$ \\
\hline $14 \mathrm{GHz}$ & $47.9+20.5$ & $42+\mathrm{j} 55.6$ \\
\hline $16 \mathrm{GHz}$ & $47.9+\mathrm{j} 20.5$ & $42+\mathrm{j} 55.6$ \\
\hline $18 \mathrm{GHz}$ & $49+\mathrm{j} 13.5$ & $35.3+\mathrm{j} 58.4$ \\
\hline
\end{tabular}

The drain and gate bias circuitries were designed using rectangular coplanar inductors in order to satisfy wideband requirement.

The empirical adjustments of bias lines are verified using electromagnetic simulations with the realized bypass capacitances.

Gain flatness is the main goal of our amplifier so in addition to gain equalization network, input and output matching topology is optimized for this purpose. The aim for this amplifier is to achieve small signal gain of $7 \pm 1 \mathrm{~dB}$ for $6 \mathrm{GHz}$ $18 \mathrm{GHz}$ frequency band

While designing the amplifier that covers broadband frequency range, multi-section matching elements have been achieved with the coplanar components.

The output matching network was designed by taking consideration into load-pull simulation data at discrete frequencies over the whole band using five section band-pass Chebyshev-type matching circuitry. Also, the output matching network utilizes from multi-section components like rectangular inductors and open stub CPW lines. Open circuit CPW lines are used instead of MIM capacitors due to better matching performance at frequency of $18 \mathrm{GHz}$. Although it is possible to use short circuited stubs instead of coplanar rectangular inductors in output matching circuitry, it is not preferred since it affects the broadband response negatively.

The input matching circuit was realized by supplying a simpler and minimized matching topology using reactive matching circuit with gain equalization network. Similar to the output matching network, shunt capacitances are realized as open stub CPW line instead of MIM capacitors. The MIM capacitances are used as DC block capacitances.

The RF performance of the MMIC amplifier was simulated accurately using Momentum simulation tool of Agilent's Advanced Design System (ADS).

Fig. 3 is a photograph of our fabricated GaN MMIC amplifier. The chip size of the device is $3.5 \mathrm{~mm} \times 1.5 \mathrm{~mm}$.

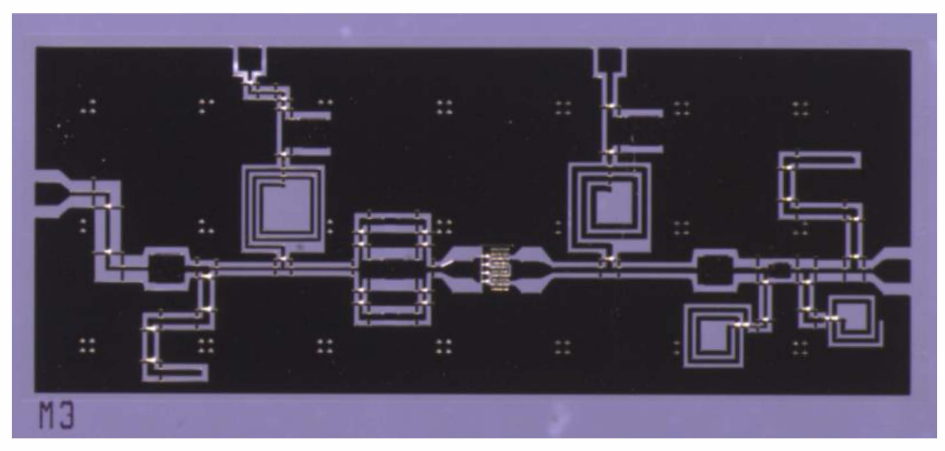

Fig. 3. Photograph of the GaN MMIC

\section{AMPLIFIER PERFORMANCE}

The small-signal response of the amplifier by comparing with the simulated small-signal gain (S21) is shown in Fig. 4. In both simulation and measurement drain voltage of the amplifier is taken as $15 \mathrm{~V}$. The design goal for the small signal gain was $7 \pm 1 \mathrm{~dB}$. The measured small signal gain varies between $6.3 \mathrm{~dB}$ and $7.5 \mathrm{~dB}$ within the entire frequency band. We have demonstrated that the resulting device gives compatible outcomes with the simulated small signal gain, input and output return losses.

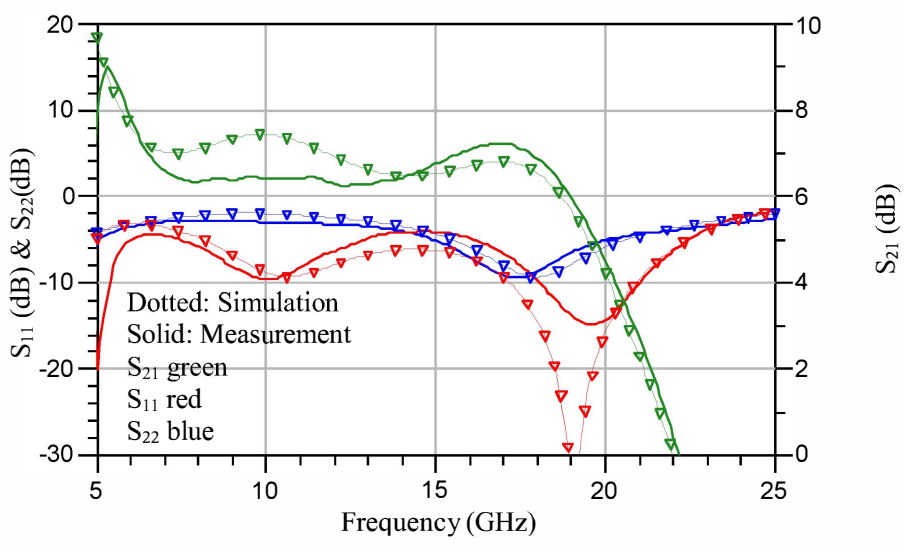

Fig. 4. S- parameters $\left(\mathrm{S}_{21}\right.$ (green), $\mathrm{S}_{11}$ ( red) and $\mathrm{S}_{22}$ (blue)) from the simulation (dotted line) and form measurement (solid line) of the MMIC 
ADS harmonic balance simulation results of the amplifier under different input power conditions for $6 \mathrm{GHz}, 8 \mathrm{GHz}, 10$ $\mathrm{GHz}, 12 \mathrm{GHz}, 14 \mathrm{GHz}, 16 \mathrm{GHz}$ and $18 \mathrm{GHz}$ are given Fig. 5 . As can be seen, saturated $\mathrm{CW}$ power is above $22 \mathrm{dBm}$ for all frequencies.

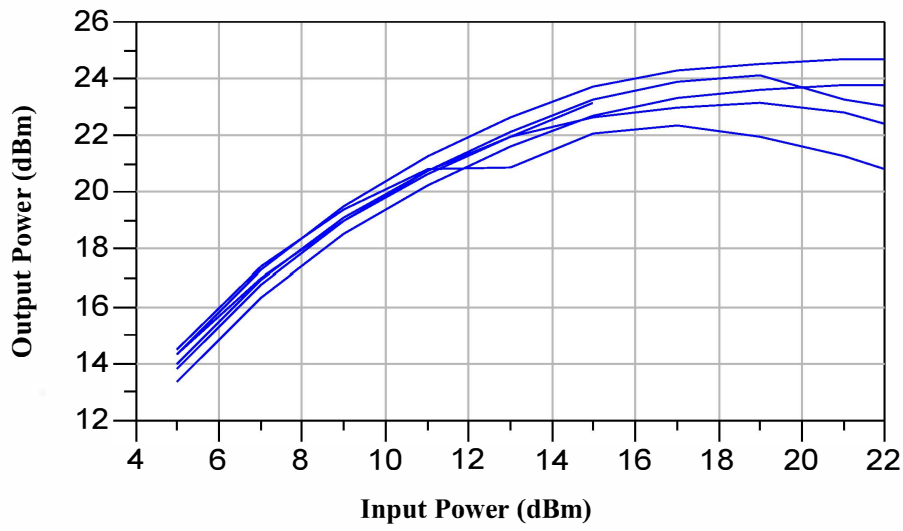

Fig. 5. Simulated CW power vs input power at different frequencies

Fig.6 shows output power and efficiency for the swept CW input power of the MMIC for different frequencies. Output power of $24 \mathrm{dBm}$ at $3 \mathrm{~dB}$ compression point was achieved over intended frequency. The measurement results are even 1$2 \mathrm{~dB}$ better than the simulated output power results.Fig.7 shows measurement results of the large signal performance of

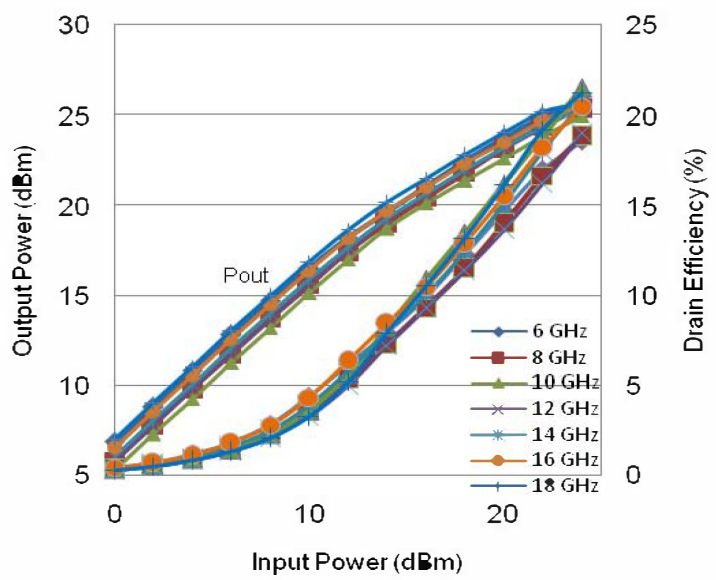

Fig. 6 Measured CW output power and drain efficiency vs input power at different frequencies

the MMIC over the band of interest using 50\% pulsed signal source. The pulsed output power larger than $27 \mathrm{dBm}$ can be obtained over the 6 to $18 \mathrm{GHz}$ band at $3 \mathrm{~dB}$ compression point with $47 \%$ peak drain efficiency when biased at $15 \mathrm{~V}$. Since the temperature rise is not as much as in $\mathrm{CW}$ measurement, higher output power levels are achieved and pulsed mode of operation is required for most of the applications. This measurement also shows that if temperature rise is controlled in $\mathrm{CW}$ mode, higher output powers can be obtained.

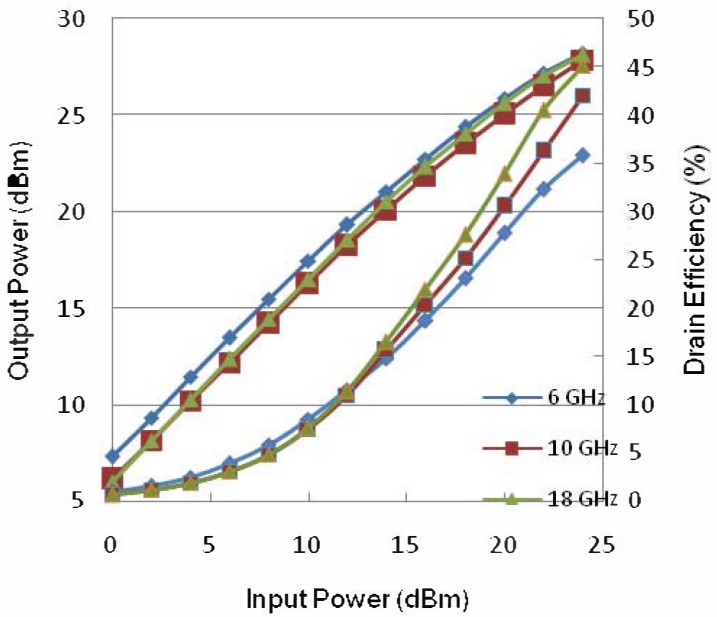

Fig. 7. Measured pulsed output power and drain efficiency vs input power at different frequencies

\section{CONCLUSION}

Using a $0.3 \mu \mathrm{m}$ GaN HEMT technology, a multi-octave band GaN-HEMT MMIC amplifier has been realized with reactively matching topologies. For the 6-18 GHz band, measurement results demonstrate a small signal gain of $7 \pm$ $0.75 \mathrm{~dB}$ and minimum $500 \mathrm{~mW}$ output power for $3 \mathrm{~dB}$ compression point in pulsed mode with related power added efficiency above $35 \%$.

The measured performances of the realized MMICs are in good agreement with electromagnetic simulation predictions.

\section{ACKNOWLEDGEMENT}

The authors would like to acknowledge Ayca Emen, Burak Turhan, Dogan Yilmaz, and Huseyin Cakmak for valuable fabrication support.

\section{REFERENCES}

[1] E. Reese, D. Allen, C. Lee, and T. Nguyen, "Wideband power amplifier MMICs utilizing GaN on SiC," 2010 IEEE MTT-S International Microwave Symposium Digest, pp. 1230 - 1233, June 2002 .

[2] U. K. Mishra, P. Parikh, and Y. Wu, "AlGaN/GaN HEMTs- An Overview of Device Operation and Applications," Proceedings of IEEE, vol. 90, no. 6, pp. 1022-1031, June 2002

[3] J.W. Milligan, S. Sheppard, W. Pribble, Y.-F. Wu, St.G. Muller, and J.W. Palmour, "SiC and GaN Wide Bandgap Device Technology Overview," IEEE Radar Conference, pp.960-964, April 2007.

[4] F. Yamada, S. Mizuno, H. Yamamoto, S. Sano, “A 6 GHz-15 GHz 2 Watts GaN MMIC," Proceedings of APMC 2011, pp. 1554-1557, December 2011.

[5] F. van Ray, R.Quay, R. Kiefer, F. Benkhelifa, B. Raynor, W. Pletschen, M. Kuri, H. Massler, S. Müller, M. Dammann, M. Mikulla, M. Schlechtweg "A Coplanar X-band $\mathrm{AlGaN} / \mathrm{GaN}$ Power Amplifier MMIC on s.i. SiC Substrate," IEEE Microwave and Wireless Component Letters, vol. 15, pp. 460-462. 